Editorial

\title{
Proteomics and bioinformatics in metabolic engineering
}

\section{Editorial}

Metabolic engineering is a developing field of biological science with a promising future. Biosynthetic pathways can nowadays be altered or introduced into bacterial, plant and animal cells and organisms. Current bioinformatics data available of total gene expression, as well as a cell proteome, help with the reconstruction of enzyme pathways for both fundamental and applied research.

While data on protein-protein interaction provide the information on the components of pathways, the regulation of their activity is a subject of a more specific research. General proteomic analysis of the "whole cell" or its fraction does not always tell if the proteins are organized in complexes, and especially how those complexes may act depending of their assembly or localization. A regulation of the quality and the amount of metabolites, produced by the pathway, may depend greatly on those factors.

How much does one have to know to successfully reconstruct or manipulate a biosynthetic pathway to achieve a desired amount of metabolites? In one of the recent reviews on a biosynthetic pathway in plants, responsible for the production of carotenoids which are antioxidants and vitamin A precursors in human body, we suggest that localization and protein complex organization play a critical role for the pathway output. ${ }^{1}$ While a generalized pathway of plant carotenoid biosynthesis can be found online through bioinformatics resources such as PlantCyc, ${ }^{2}$ a paper-written scheme is not enough to understand the mechanisms of control of its activity. We found that in many plants one of the most important enzymes of this pathway, the phytoene synthase, exists in a form of several isozymes, which can potentially form different complexes, thus affecting amounts of carotenoids produced. ${ }^{3}$ Sufficient amount of carotenoids in plant food is important to satisfy our nutritional needs, so the research on biosynthetic pathway protein complexes is necessary to provide information for pathway altering or breeding of existing plant varieties.

In order to uncover the intra-cellular localization of enzyme complexes and the identity of the complete pathway metabolon, current proteomic methods are a great help. Cell fractionation, native protein complexes isolation and peptide sequencing allow separating of a very specific membrane compartment, isolating protein complexes under non-denaturing conditions and identifying the exact members
Volume I Issue 4 - 2014

\author{
Maria Shumskaya \\ Department of Biological Sciences, Lehman College, The City \\ University of New York (CUNY), USA
}

\author{
Correspondence: Maria Shumskaya, Department of Biological \\ Sciences, Lehman College, The City University of New York \\ (CUNY), Bronx, New York, 10468, USA, \\ Email maria.shumskayaI@lehman.cuny.edu
}

Received: August 08, 2014 | Published: August 09, 2014

of metabolons with LC MS/MS techniques. Quadruple mass analyzer with accuratemass detection can deliver exceptional performance in identifying peptides in a mixture of several proteins, such as an enzyme complex, isolated either by chromatography or a native gel without complicated 2D techniques. Hopefully, more research will be done to reveal multidimensional srtuctures of different enzyme pathways using proteomic techniques to fulfill our knowledge on metabolon performance.

I wish MOJ Proteomics and Bioinformatics becomes home for the research on the protein components of metabolic pathways and serve a great platform to the future endeavors in metabolic engineering.

\section{Acknowledgements}

None.

\section{Conflict of interest}

The author declares no conflict of interest.

\section{References}

1. Shumskaya M, Wurtzel ET. The carotenoid biosynthetic pathway: thinking in all dimensions. Plant Sci. 2013;208:58-63.

2. http://pmn.plantcyc.org/PLANT/NEW-IMAGE?type=PATHWAY\&obje $\mathrm{ct}=$ CAROTENOID-PWY

3. Shumskaya M, Bradbury LM, Monaco RR, et al. Plastid localization of the key carotenoid enzyme phytoene synthase is altered by isozyme, allelic variation, and activity. Plant Cell. 2012;24(9):3725-3741. 